\title{
Clonal chromosome anomalies affecting FLI1 mimic inherited thrombocytopenia of the Paris-Trousseau type
}

\author{
Patrizia Noris ${ }^{1}$, Roberto Valli ${ }^{2}$, Alessandro Pecci ${ }^{1}$, Cristina Marletta ${ }^{2}$, Rosangela Invernizzi ${ }^{1}$, Lydia \\ Mare $^{2}$, Carlo L. Balduini ${ }^{1}$, Emanuela Maserati ${ }^{2}$ \\ ${ }^{1}$ Istituto di Ricovero e Cura a Carattere Scientifico Policlinico San Matteo Foundation, University of Pavia, Pavia, Italy; ${ }^{2}$ Dipartimento di Medicina \\ Clinica e Sperimentale, Università dell'Insubria, Varese, Italy
}

\begin{abstract}
Introduction: The thrombocytopenia of the Paris-Trousseau (TCPT) type is a contiguous gene syndrome characterized by mild bleeding tendency, variable thrombocytopenia (THC), abnormal giant alpha-granules in platelets and dysmegakaryopoiesis: it derives from a constitutional deletion of chromosome 11 leading to the loss of $F L / 1$, a transcription factor involved in megakaryocyte differentiation and maturation. Case report: A women with an acquired, isolated THC developing over $10 \mathrm{yr}$ showed morphological features typical of TCPT in platelets and bone marrow (BM). Twenty years after the onset of THC, the other hematological parameters are still normal and the patient is well. Results: Clonal hemopoiesis was shown and chromosome analyses performed on BM revealed a clone with 45 chromosomes and a complex unbalanced translocation involving chromosomes 2, 3, and 11. The anomaly was present in the majority of bone marrow cells but only in a few peripheral blood elements. A microarray-based comparative genomic hybridization defined the deleted region of chromosome 11 including the FL/1 locus that was missing. Conclusion: Although our patient presented with nearly all the characteristics of TCPT, her illness was acquired instead of being inherited and the most appropriate diagnosis is that of the unilineage dysplasia 'refractory THC.' This observation suggests that appropriate cytogenetic investigations should be always considered in patients with acquired THC of unknown origin.
\end{abstract}

Key words acquired thrombocytopenia; FLI1; thrombocytopenia Paris-Trousseau type

Correspondence Patrizia Noris, Clinica Medica III, Fondazione IRCCS Policlinico San Matteo, Università di Pavia, Piazzale Golgi, 27100 Pavia, Italy. Tel: 0039-0382-502688; Fax: 0039-0382-526223; e-mail: p.noris@smatteo.pv.it

PN and RV contributed equally to this manuscript.

Accepted for publication 2 July 2012

doi:10.1111/j.1600-0609.2012.01833.x

FLII is a member of the ETS family of transcription factors. It trans-activates several genes involved in megakaryocyte (Mk) differentiation and maturation, as ITGA2 (1), GP9 (2), GPIBA (3), and $c-m p l$ (4), and its targeted disruption in mice results in a dramatic reduction in MKs number and size $(5,6)$. Subtle constitutional deletions of the long arms of chromosome 11, including the FLIIlocus, causes the Paris-Trousseau syndrome (TCPT, OMIM \# 188025) (7), a thrombocytopenia with abnormal giant alpha-granules in platelets, whereas larger deletion imply a variety of additional phenotype changes, often recognized as Jacobsen syndrome (OMIM \# 147791) (8). Thus, defective FLII is one of the many causes of inherited thrombocytopenia that is considered in the differential diagnosis of these disorders.

We report here the first patient with an acquired form of thrombocytopenia associated with a clonal chromosome anomaly in the bone marrow (BM) with the loss of FLII that leads to the picture of TCPT.

\section{Patient and methods}

\section{Case report}

An Italian young woman, born in 1972 from non-consanguineous parents and with irrelevant family history, was 
referred for thrombocytopenia. She had undergone a left nephrectomy for congenital hydronephrosis at the age of $11 \mathrm{yr}$. Since then she made regular blood tests that showed a progressive reduction in platelet count (Fig. 1), while the other blood cells were always within normal limits. At the age of $23 \mathrm{yr}$, her platelet count fell steadily below $100 \times 10^{9} / \mathrm{L}$, and she was diagnosed with autoimmune thrombocytopenia. At 32, her platelet count was lower than $50 \times 10^{9} / \mathrm{L}$ and she received prednisone $1 \mathrm{mg} / \mathrm{Kg} / \mathrm{d}$ for 3 months without any benefit. Soon after, BM examination revealed an increased number of megakaryocytes (Mks), often small. At 36, administration of intravenous immunoglobulins $(0.4 \mathrm{~g} / \mathrm{kg} / \mathrm{d}$ for $5 \mathrm{~d})$ did not increase platelet count.

Examined for the first time at our institution in 2009, at the age of 37, she had no bleeding tendency. Blood count showed: Hb $15.6 \mathrm{~g} / \mathrm{dL}, \mathrm{MCV} 82.9 \mathrm{fL}, \mathrm{WBC} 5.5 \times 10^{9} / \mathrm{L}$ (64.5\% neutrophils, $24 \%$ lymphocytes, $4 \%$ monocytes, $7 \%$ eosinophils, and $0.5 \%$ basophils), platelets $42 \times 10^{9} / \mathrm{L}$, MPV $15.8 \mathrm{fL}$. In vitro platelet aggregation, measured by the densitometric method of Born after stimulation with collagen $4 \mu \mathrm{g} / \mathrm{mL}$, ADP $5 \mu \mathrm{M}$ and ristocetin $1.5 \mathrm{mg} / \mathrm{mL}$ was within the normal range (data not shown).

Evaluation of May-Grünwald-Giemsa (MGG) stained peripheral blood (PB) films did not identify red cell or leukocyte abnormalities, but it showed platelet macrocytosis with some elements larger than red cells (giant platelets). Moreover, a few platelets $(0.3 \%)$ had only one, large, azurophilic granule (Fig. 2). Immunofluorescence microscopy (9) revealed that these giant granules contained thrombospondin 1 (TSP1) and platelet factor 4, thus indicating that they were abnormally large alpha-granules (Fig. 2). To better define their morphologic features, we performed electron microscopy (EM) study of peripheral blood platelets, but we failed to identify abnormally large granules, probably because of their extreme rarity and the difficulty of examining a sufficient number of cells by this technique.

MGG-stained BM touch preparations displayed normocellular marrow with markedly increased, often clustered, Mks. The numerous Mks were dysplastic with prevalence of

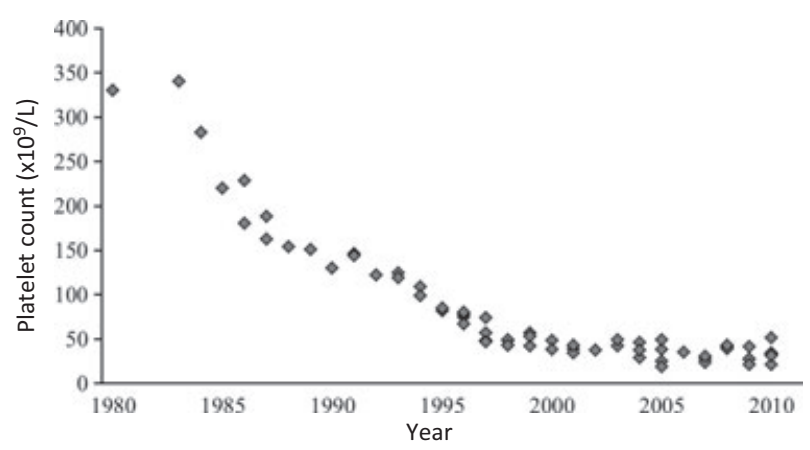

Figure 1 Time course of platelet count. A progressive reduction in platelet count began when the patient was 12 -yr old and led to a severe thrombocytopenia with $<50 \times 10^{9} / \mathrm{L}$ platelets in about $15 \mathrm{yr}$. mononuclear micromegakaryocytes, small binucleate Mks, and Mks with agranular or vacuolated cytoplasm (Fig. 2). Erythroid and granuloblastic lineages were morphologically normal with $3 \%$ blasts and slightly increased eosinophils. PERLS's staining demonstrated the absence of ring sideroblasts among erythroid precursors (not shown).

Serum thrombopoietin (TPO) level, evaluated as previously reported (10), was within the normal range (29.7 pg/ mL, n.v. 6.9-54.4). Evaluation of clonality with the method of HUMARA in peripheral blood neutrophils revealed clonal hemopoiesis (11).

At the last evaluation (June 20, 2012), the patient's clinical condition was unchanged and the only alteration of blood count was still a moderate thrombocytopenia $\left(51 \times 10^{9}\right.$ platelets/L).

The Ethic Committee of the IRCCS San Matteo Hospital Foundation approved this study and the investigated patient gave written informed consent.

\section{Cytogenetic methods}

Chromosome analyses were performed with routine methods and QFQ-banding technique on BM direct preparations and 24-48 h cultures, on PB unstimulated and phytohaemagglutinin (PHA)-stimulated cultures. Fluorescence in situ hybridization (FISH) was used to better define the complex structural anomaly found in the BM with multipainting technique with the 24XCyte-MetaSystems' 24 color kit (Metasystems GmbH, Althussheim, Germany); the BAC probe RP11-744N12, which recognizes a sequence in band q24.3 of chromosome 11, including the gene FLII, was used for FISH on BM mitoses and interphase nuclei, and BAC probes RP11-1152N24 (2q36.1) and CTC-774G23 (3p12.3) were used in double color FISH (Invitrogen Corporation, Carlsbad, CA, USA).

Microarray-based comparative genomic hybridization (a-CGH) with the $244 \mathrm{~K}$ genome-wide system (Agilent Technologies Inc., Santa Clara, CA, USA) was performed according to the manufacturer's instruction on DNA from BM. The DNA was extracted using the Qiagen Blood and Tissue Kit (QIAGEN GmbH, Hilden, Germany), and competitor DNA was purchased from Promega (Madison, WI, USA). Slides were scanned using Agilent's microarray scanner G2565CA and microarray images were analyzed using Agilent's Feature Extraction 10.7.3.1 software, and by Agilent's Genomic Workbench software (5.0.14). All map positions in the results refer to the genome assembly hg18.

\section{Results}

Chromosome analyses performed on BM in November 2008 and October 2009 revealed a clone with 45 chromosomes and a complex structural anomaly involving chromosomes 2 , 3 , and 11. It was interpreted as an unbalanced complex 

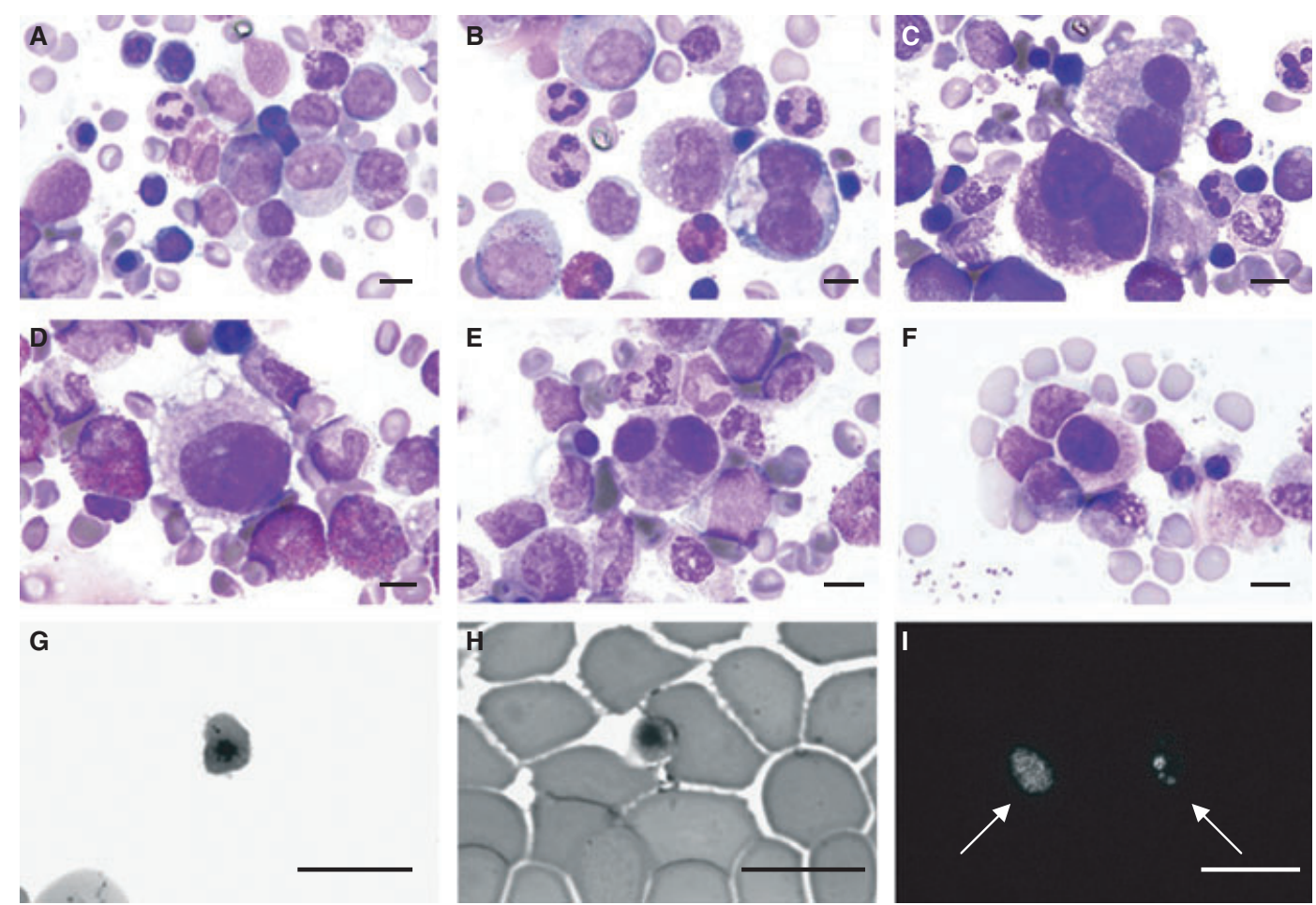

Figure 2 (A-F) BM touch preparation. BM smears showed normal erythroid and granulocytic cells (A) and evident dysmegakaryopoiesis. (B) An immature Mk with vacuolated cytoplasm. (C) A Mk with hypogranular cytoplasm (top right); the other Mk looks normal (bottom left). (D) A large Mk with a single large round nucleus and hypogranular vacuolated cytoplasm. (E) A small binucleate Mk. (F) A micromegakaryocyte with scanty granular cytoplasm. (G-I) Peripheral blood film, black and white. Rare platelets with a single large granule are observed in MGG-stained film (G and H). Immune-staining for TSP1 of peripheral blood films (I): the platelet on the left has normal alpha-granule content and distribution, while that on the right have one prominent, large granule. Scale bars correspond to 10 micronmeters.

translocation, leading to partial monosomy of the long arms of chromosome 2, of the pericentromeric region (short and long arms) of chromosome 3, and of the long arms of chromosome 11 . The anomaly was present in the majority of the cells, together with normal cells, with identical proportions at the two times of analysis: 45,XX, $\operatorname{der}(2) \mathrm{t}(2 ; 3)(\mathrm{q} 36.1 ; \mathrm{p} 12.3)$,$3, \operatorname{der}(11) \mathrm{t}(3 ; 11)(\mathrm{q} 11.2 ; \mathrm{q} 14.3)[39] / 46, \mathrm{XX}[1]$. The same abnormal clone was detected in one single cell from PB unstimulated culture in October 2009, and in PHA-stimulated cultures (2/40 mitoses in June 2009, and 2/31 in October 2009).

The multipainting technique and the FISH results with the BAC probes confirmed the results of the chromosome analyses, and a-CGH on BM (October 2009) precisely defined the regions of imbalance, which may be described by the following formulation: arr2q36.1qter (221 $960583-$ 242689 978) $\times 1, \quad 3 p 12.3 p 11(74438$ 852-90 391698$) \times 1$, 11q14.3qter(91 175 044-134 432 265) × 1, (Fig. 3). On chromosome 3, a-CGH revealed the short arms monosomy, whereas no imbalance of the long arms was put in evidence, because the breakpoint was close to the centromere, and the oligonucleotides spotted on the a-CGH platform did not cover this region. In particular, the deleted region of chromosome 11 included the FLII locus that was therefore missing: FISH with the probe RP11-744N12 confirmed its loss on mitoses and on BM interphase nuclei (200 nuclei out of 313 in October 2009).

\section{Discussion}

TCPT is a rare contiguous gene syndrome because of a constitutional interstitial deletion or microdeletion of the long arms of chromosome 11, with the loss of one allele of the gene FLII (7), in some cases with apparent autosomal dominant inheritance. As in many inherited thrombocytopenias, blood platelets are typically larger than normal, but a distinguishing feature of TCPT is that a little percentage of circulating platelets has one or more giant granules that derive from the fusion of alpha-granules. Another characteristic finding of TCPT is the abundance of BMMks, which appear severely dysplastic, with many small and immature forms.

Our patient presented with nearly all the hematological characteristics of TCPT. She had both macrothrombocytopenia and some platelets with giant alpha-granules, a greatly increased number of BM Mks, with most of them showing clearly dysplastic features, and she carried a complex 

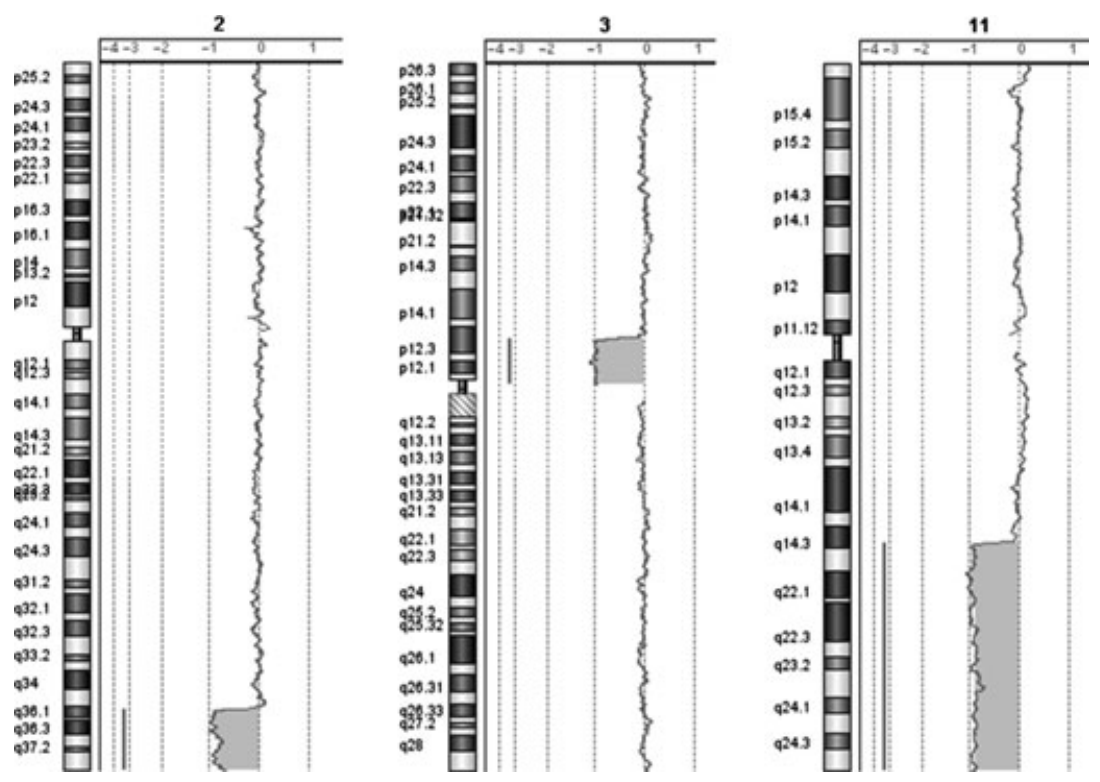

Figure 3 a-CGH profiles of chromosomes 2, 3, and 11 showing the unbalanced regions.

chromosome structural anomaly including the loss of one FLII allele. As this chromosome defect affected most of the BM cells but only a very limited proportion of peripheral blood elements, we can conclude that it was acquired in $\mathrm{BM}$, instead of being constitutional as in the TCPT patients reported so far: consequently, the disease was not congenital, but gradually developed over a period of more than $20 \mathrm{yr}$. The positivity of the assay for clonal hematopoiesis agrees with cytogenetic data. An alternative, more convincing diagnosis for our patients is 'refractory thrombocytopenia', a form of myelodysplastic syndrome (MDS) that, together with 'refractory anemia' and 'refractory neutropenia,' constitutes the category of 'refractory cytopenias with unilineage dysplasia', as defined in the 2008 revision of the World Health Organization classification of myeloid neoplasms and acute leukemias (12). Our patient meets all the criteria for refractory thrombocytopenia: she acquired an isolated thrombocytopenia with $<100 \times 10^{9}$ platelets/L and had no blasts in peripheral blood; more than $10 \%$ of BMMks had clearly dysplastic features; no erythroid precursors were ring sideroblasts and $\mathrm{BM}$ blasts were $<5 \%$. What is puzzling in this MDS patient is the very prolonged clinical course of her illness: although platelet count fell below $150 \times 10^{9} / \mathrm{L}$ more than $20 \mathrm{yr}$ ago and below $100 \times 10^{9} / \mathrm{L} 17 \mathrm{yr}$ ago, thrombocytopenia continues to be the only cytopenia, because both the value of $\mathrm{Hb}$ and the number of neutrophils are still within the normal limits. Also taking into account that thrombocytopenia was identified at its first appearance because the patient was accustomed to carry out regular blood tests, an event-free survival longer than $20 \mathrm{yr}$ is surprising, because much shorter clinical courses have been described for refractory thrombocytopenias reported so far $(13,14)$. According to the International Scoring System for Evaluating Prognosis in Myelodysplastic Syndromes (15), our patient would have an intermediate-1 risk and an age-related expected survival of $5.2 \mathrm{yr}$. Also, in this respect, the evolution of the disease was therefore much slower than expected.

The trend of platelet count described in Fig. 1 seems to indicate that, after a 15 -yr-long gradual descent, the number of platelets has remained fairly stable over the last $10 \mathrm{yr}$. We may speculate that platelet count decreased while the abnormal BM clone with defective FLII was expanding, and that the degree of thrombocytopenia remained subsequently stable because no other genetic defects hampering megakaryopoiesis occurred. Based on these considerations, we believe that the diagnosis of a refractory thrombocytopenia that mimics TCPT because of the loss of one FLII allele is appropriate for this patient. Our observation emphasizes that every patient with MDS is a story unto itself and that the prognostic indicators should be used with caution in making treatment decisions. In particular, the complex genetic defect of our patient was compatible with a very long survival without any severe clinical defects despite the poor prognosis indicated by the IPSS prognostic index.

In conclusion, the patient we described here acquired a MDS that has posed problems of differentiation not only from immune thrombocytopenia but also from the TCPT. We believe, therefore, that this rare form of MDS should be considered in the differential diagnosis of all patients with acquired thrombocytopenias and occasional giant platelet alpha-granules. Being a clonal chromosome aomaly the primary event leading to thrombocytopenia in the patient reported here, we suggest that cytogenetic investigations, including a-CGH, should always be considered in the 
diagnostic evaluation of patients with acquired thrombocytopenia of unknown origin with the clinical picture of our patient.

\section{Acknowledgements}

Supported by Fondazione Banca del Monte di Lombardia and of Associazione Italiana Sindrome di Shwachman (AISS) to FP and EM. Partly supported by grants from Ministero dell'Istruzione, dell'Università e della Ricerca to PN, $\mathrm{AP}$, and $\mathrm{CB}$.

\section{References}

1. Lemarchandel V, Ghysdael J, Mignotte V, Rahuel C, Roméo PH. Gata and Etscis-acting sequence mediate megakaryocytespecific expression. Mol Cell Biol 1993;13:668-76.

2. Bastian LS, Kwiatkowski BA, Breininger J, Danner S, Roth G. Regulation of the megakaryocytic glycoprotein IX promoter by the oncogenic Ets transcription factor Fli-1. Blood 1999;93:2637-44.

3. Hashimoto Y, Ware J. Identification of essential GATA and Ets binding motifs within the promoter of the platelet glycoprotein Ib $\alpha$ gene. J Biol Chem 1995;270:24532-9.

4. Deveaux S, Filipe A, Lemarchandel V, Ghysdael J, Roméo $\mathrm{PH}$, Mignotte V. Analysis of the thrombopoietin receptor (MPL) promoter implicates GATA and Ets proteins in the coregulation of megakaryocyte-specific genes. Blood 1996;87:4678-85.

5. Hart A, Melet F, Grossfeld P, Chien K, Jones C, Tunnacliffe A, Favier R, Bernstein A. Fli-1 is required for murine vascular and megakaryocytic development and is hemizygously deleted in patients with thrombocytopenia. Immunity 2000; 13:167-77.

6. Spyropoulos DD, Pharr PN, Lavenburg KR, Jackers P, Papas TS, Ogawa M, Watson DK. Hemorrhage, impaired hematopoiesis, and lethality in mouse embryos carrying a targeted disruption of the Fli1 transcription factor. Mol Cell Biol 2000;20:5643-52.
7. Favier R, Jondeau K, Boutard P, Grossfeld P, Reinert P, Jones C, Bertini F, Cramer EM. Paris-Trousseau syndrome: clinical, haematological, molecular data of ten new cases. Thromb Haemost 2003;90:893-7.

8. Van Zutven LJ, van Bever Y, Van Nieuwland CC, Huijbregts GC, Van Opstal D, von Bergh AR, Corel LJ, Tibboel D, Wouters $\mathrm{CH}$, Poddighe PJ. Interstitial 11q deletion derived from a maternal ins $(4 ; 11)(\mathrm{p} 14 ; \mathrm{q} 24.2 \mathrm{q} 25)$ : a patient report and review. Am J Med Genet 2009;149A:1468-75.

9. De Candia E, Pecci A, Ciabattoni G, De Cristoforo R, Rutella S, Yao-Wu Z, Lazzareschi I, Landolfi R, Coughlin S, Balduini CL. Defective platelet responsiveness to thrombin and protease-activated receptors agonists in a novel case of gray platelet syndrome: correlation between the platelet defect and the alpha-granule content in the patient and four relatives. J Thromb Haemost 2007;5:551-9.

10. Noris P, Perrotta S, Seri M, et al. Mutations in ANKRD26 are responsible for a frequent form of inherited thrombocytopenia: analysis of 78 patients from 21 families. Blood 2011;117:6673-80.

11. Balduini CL, Pecci A, Loffredo G, et al. Effects of the R216Q mutation of GATA-1 on erythropoiesis and megakaryocytopoiesis. Thromb Haemost 2004;91:129-40.

12. Vardiman JW, Thiele J, Arber DA, et al. The 2008 revision of the World Health Organization (WHO) classification of myeloid neoplasms and acute leukemia: rationale and important changes. Blood 2009;30:937-51.

13. Breccia M, Latagliata R, Cannella L, Carmosino I, Santopietro M, Loglisci G, Federico V, Alimena G. Refractory cytopenia with unilineage dysplasia: analysis of prognostic factors and survival in 126 patients. Leuk Lymphoma 2010;51:783-8.

14. Marinier DE, Mesa H, Rawal A, Gupta P. Refractory cytopenias with unilineage dysplasia: a retrospective analysis of refractory neutropenia and refractory thrombocytopenia. Leuk Lymphoma 2010;51:1923-6.

15. Greenberg P, Cox C, LeBeau MM, et al. International scoring system for evaluating prognosis in myelodysplastic syndromes. Blood 1997;89:2079-88. 